\title{
qSOFA score for prediction of sepsis outcome in emergency department
}

\author{
Kavous Shahsavarinia ${ }^{1}$, Payman Moharramzadeh², \\ Reza Jamal Arvanagi $i^{3}$ Ata Mahmoodpoor ${ }^{4}$
}

\begin{abstract}
Objective: The third international consensus definition for sepsis and septic shock (sepsis 3) task force recently introduced qSOFA (quick sequential organ failure assessment) as a score for detection of patients at risk of sepsis outside of intensive care units. We performed this study to evaluate the validity of qSOFA for early detection and risk stratification of septic patients in emergency department.

Methods: We conducted this study in an emergency department of the largest university affiliated hospital in northwest of Iran from Sept 2015 to Sept 2016. One hundred and forty patients who were SIRS positive with a suspected infection without alternative diagnosis and a microbiological proven infection were enrolled in this study. qSOFA was calculated for each patient and correlated with sepsis grades and mortality.

Results: From 140 patients $84(60 \%)$ had positive qSOFA score and 56 (40\%) patients had negative qSOFA score. Our results showed that near half of patients with positive qSOFA expired during their stay in hospital while this was about $5 \%$ for patients with negative qSOFA. ROC curve of study regarding prediction of outcome with qSOFA showed an area under curve of 0.59. ( $P$ value: 0.04$)$. Time spent to sepsis detection was 16 minutes shorter with qSOFA score compared to SIRS criteria in this study.

Conclusion: In patients with suspected sepsis, qSOFA has acceptable value for risk stratification of severity, multi organ failure and mortality. It seems that education of medical staff and frequent screening of patients for warning signs can help to increase the value of qSOFA in prediction of mortality in critically ill septic patients.
\end{abstract}

KEYWORDS: Emergency department, qSOFA (quick sequential organ failure assessment), Sepsis.

How to cite this:

doi: https://doi.org/10.12669/pjms.36.4.2031

Shahsavarinia K, Moharramzadeh P, Arvanagi RJ, Mahmoodpoor A. qSOFA score for prediction of sepsis outcome in emergency department. Pak J Med Sci. 2020;36(4):668-672. doi: https://doi.org/10.12669/pjms.36.4.2031

This is an Open Access article distributed under the terms of the Creative Commons Attribution License (http://creativecommons.org/licenses/by/3.0), which permits unrestricted use, distribution, and reproduction in any medium, provided the original work is properly cited.

1. Kavous Shahsavarinia,

Associate Professor,

Road Traffic Injury Research Center,

2. Payman Moharramzadeh,

Associate Professor,

3. Reza Jamal Arvanagi,

Emergency Medicine Specialist,

4. Prof. Dr. Ata Mahmoodpoor,

Dept. of Anesthesiology and intensive care, Faculty of Medicine,

1-3: Department of Emergency Medicine, Faculty of Medicine,

1-4: Tabriz University of Medical Sciences, Tabriz, Iran.

Correspondence:

Prof. Dr. Ata Mahmoodpoor, MD, FCCM.

General ICU, Shohada Hospital,

El-Goli Street, Tabriz, Iran.

Email: amahmoodpoor@yahoo.com

* Received for Publication:

November 29, 2019

* $1^{\text {st }}$ Revision Received:

* $2^{\text {nd }}$ Revision Received:

* Final Revision Accepted:
December 2, 2019

February 22, 2020

February 25, 2020

\section{INTRODUCTION}

Sepsis is a huge global health problem and represents great challenge regarding diagnosis and treatment for physicians. ${ }^{1}$ Sepsis is a frequently encountered life threatening condition and is estimated that approximately eight hundred fifty thousand (850000) cases of sepsis visit emergency department annually which is associated with high cost. $^{2}$ Sepsis is defined as life threatening organ dysfunction caused by dysregulated host response to infection. ${ }^{3}$ Organ dysfunction is characterized by acute increase in two or more points of sequential organ failure assessment (SOFA) score; but as it requires different tests and rarely measured outside the ICU, a new score, qSOFA (quick 
sequential organ failure assessment), is introduced by sepsis task force. ${ }^{3}$

Results of a recently performed cohort study showed that qSOFA was better than SIRS in predicting in-hospital mortality in septic patients in emergency department. ${ }^{4}$ On the other hand, data from New Zealand intensive care society showed that interventions like intubation, sedation and mechanical ventilation can interfere with the validity and accuracy of qSOFA score in the critically ill patients. ${ }^{5,6}$ Thus, SOFA remains the only score for prediction of sepsis in this setting. Rodriguez et al.(2018) showed that qSOFA is equal or better than SIRS (systemic inflammatory response syndrome) in predicting the critical illness in patients with sepsis admitted from ED. ${ }^{7}$ Results of two recently performed studies showed that although qSOFA may be valuable in predicting sepsis related mortality but it is a poorly sensitive marker for mortality prediction as it can delay the initiation of intervention known to improve sepsis related outcomes. ${ }^{8,9}$

Based on the above, there are some criticism about low sensitivity of qSOFA for prediction of outcome and resultant delayed diagnosis of sepsis and lack of endorsement by scientific societies and resultant mis-implication as a clinical decision tool. ${ }^{10-12}$ Churpek et al.(2017) showed that commonly used early warning scores are more accurate than qSOFA in prediction of mortality in septic patients outside the ICU. ${ }^{13}$ Results of two other studies showed that qSOFA had good prognostic value for mortality in septic patients in resource limited countries and supports using it as a triage tool to identify the patients at risk of poor outcome in resource limited countries. ${ }^{14,15}$ Based on the literature, it seems that qSOFA has been found as a contradictory marker for its diagnostic and prognostic value of sepsis evaluated so far. We performed this study to evaluate the diagnostic and prognostic value of qSOFA in septic patients admitted to emergency department.

\section{METHODS}

After obtaining ethics committee approval (Ref. No.: 5/d/20365, dated April 19, 2018), one hundred and forty patients admitted to emergency department with sepsis diagnosis were included in this study. We performed a retrospective study on all patients transferred to emergency medicine department of Imam Reza hospital between $1^{\text {st }}$ Sept 2015 and $1^{\text {st }}$ Sep 2016.
All patients with a suspected infection who were SIRS positive without alternative diagnosis and a microbiological proven infection were enrolled in this study. Exclusion criteria were patients with age less than 18 years old pregnant women, prisoners, severe traumatic patients, and patients with malignancy. Suspicion of infection was made using the following data extracted from hospital records: blood pressure, heart rate, body temperature, respiratory rate and level of consciousness. Demographic and laboratory variables were recorded for all patients. We calculated qSOFA for each patient. The score ranges from 0 to three with one point allocated for each of the following clinical signs: systolic blood pressure $<100 \mathrm{mmHg}$ respiratory rate $>22$ / minutes and altered mental status from baseline. A score of equal or more than two indicates more severity with increased ICU length of stay and mortality. The SIRS criteria use the clinical criteria of surviving sepsis campaign for severe sepsis ${ }^{15}$ with the presence of at least two of the followings: $\mathrm{HR}>90 / \mathrm{min}, \mathrm{RR}>25 / \mathrm{min}$. $\mathrm{BT}>38$ or $\mathrm{BT}<36$, WBC $>12000$ or less than 4000 . We considered patients as sepsis if they had two or more than SIRS criteria with positive microbial cultures, severe sepsis if had sepsis with organ failure, septic shock if they need vasopressor to keep their systolic blood pressure more than 90 and multi organ dysfunction syndrome as septic shock with two or more organ failure. We performed an education course to all emergency department medical staff regarding qSOFA warning alarms one month before study enrollment.

Statistical analysis: All data were expressed as mean \pm standard deviation (SD) and analyzed using the Statistical Package for Social Sciences (version 20). We used students' test for comparing variables. We used ROC curve analysis for prediction of outcome in patients. Specificity and sensitivity of qSOFA for outcome prediction were also calculated. P value of $<0.05$ was considered to be statistically significant.

\section{RESULTS}

One hundred and forty patients were enrolled in this study. The mean value for their age was $59 \pm 16.1$ and $45.7 \%$ of them were women. The mean age of both sex did not have a significant difference ( $P$-value: 0.46). We divided our patients due to their severity of illness into four groups: sepsis, severe sepsis, septic shock and multi organ failure. Demographic characteristics 
Table-I: Demographic characteristics of patients.

\begin{tabular}{lc}
\hline Variable & Value \\
\hline Sex M/F & $76 / 64$ \\
Age & $59 \pm 16.1$ \\
APACHE score & $25.3 \pm 10.43$ \\
Comorbidities & \\
Diabetes & 31 \\
Hyperlipidemia & 19 \\
Hypertension & 45 \\
IHD/CHF & 38 \\
Kidney disease & 8 \\
Lung disease & 17 \\
Liver disease & 2 \\
Neurologic disorder & 9 \\
\hline
\end{tabular}

M/F: Male/Female, APACHE: Acute Physiologic and Chronic Health Evaluation. IHD/CHF: Ischemic Heart Disease/Congestive Heart Failure.

of patients are shown in Table-I. From 140 patients, $84(60 \%)$ had positive qSOFA score and 56 patients had negative qSOFA score. During the study period, 121 patients were survived and 19 patients were expired. All of patients with septic shock and multi organ failure expired during their hospitalization. Among patients with sepsis and severe sepsis $98.7 \%$ and $89 \%$ survived respectively. Our results showed that $32 \%$ of patients with positive qSOFA were expired during their stay while this was about $5 \%$ for patients with negative qSOFA (Table-II). ROC

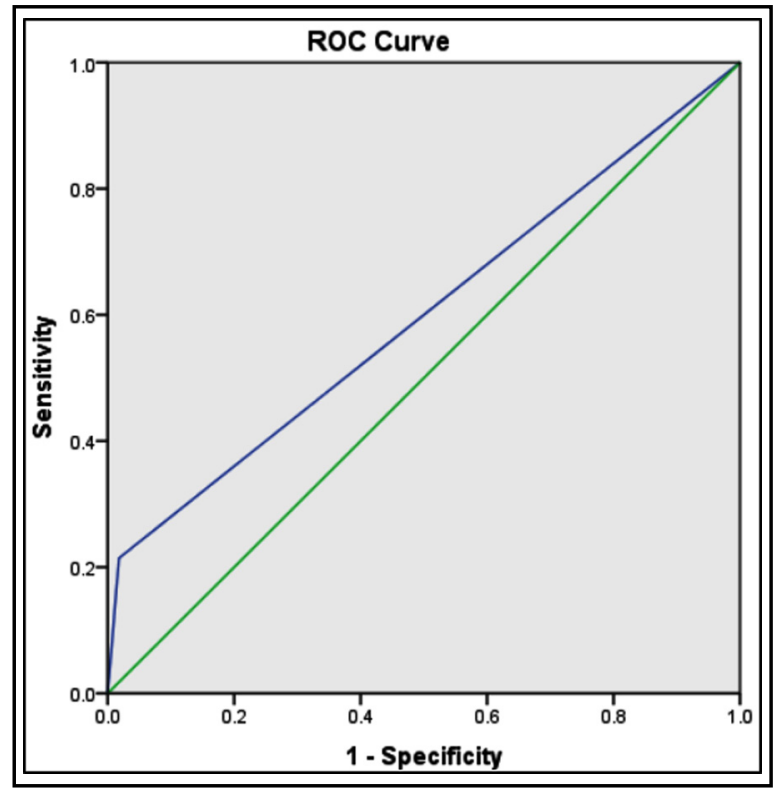

Fig.1: Prediction of outcome by qSOFA.
Table-II: qSOFA and patient's outcomes

\begin{tabular}{lc}
\hline Variable & Value \\
\hline qSOFA +/- & \\
Sepsis & $39 / 40$ \\
Severe sepsis & $32 / 16$ \\
Septic shock & $10 / 0$ \\
MOF & $3 / 0$ \\
Mean Hospital stay (day) & \\
Sepsis & $5.74 \pm 2.58$ \\
Severe sepsis & $9.36 \pm 3.89$ \\
Septic shock & $5.6 \pm 1.83$ \\
MOF & $2.66 \pm 1.52$ \\
Outcome (survived) & \\
Sepsis & $78(98.7 \%)$ \\
Severe sepsis & $43(89 \%)$ \\
Septic shock & $0(0 \%)$ \\
MOF & $0(0 \%)$ \\
\hline
\end{tabular}

curve of study regarding prediction of outcome with qSOFA showed an area under curve of 0.59. ( $P$-value: 0.04$)$ (Fig.1). In this study sensitivity of qSOFA for detection of sepsis was $66.3 \%$ with a specificity of $60.6 \%$. Negative predictive value and positive predictive value for qSOFA in sepsis detection was $35.7 \%$ and $84.5 \%$, respectively based on the clinical diagnostic statement by surviving sepsis campaign. Time spent to sepsis detection is 16 minutes shorter with qSOFA score compared to SIRS criteria in this study as this score didn't need laboratory assessment. This is performed with the time duration between suspicion of sepsis and the confirmation of diagnosis.

\section{DISCUSSION}

Results of this study showed that qSOFA score was associated with increased mortality and organ failure in septic patients. Thus, qSOFA is a good prognostic marker for mortality and multi organ failure in septic patients but is not a good diagnostic marker for sepsis detection. Our findings were similar to previous study in Malawy which showed that qSOFA is a simple tool that can aid risk stratification in resource-limited settings. ${ }^{16}$

Although there is huge evidence regarding optimal management and early detection of sepsis in ICUs, medical staff especially in emergency departments has an essential role in optimal management and early detection of these patients. ${ }^{1}$ So, all medical staff needs to know a simple and easy score to diagnose sepsis and detect the patients with poor outcome for appropriate management. ${ }^{17}$ Rudd 
et al. evaluated the validity of qSOFA in low middle income countries showed that among hospitalized patients with suspected infection, the qSOFA score compared to SIRS criteria better identified patients with high risk of death but predictive value differs among different centers/countries. ${ }^{18}$ Opposite to this results, Askim et al. ${ }^{19}$ showed that qSOFA failed to be a risk stratification tool to predict mortality in patients with severe sepsis because of low sensitivity. These results are in contrast with our results which showed only a good predictive role of qSOFA in prediction of mortality in septic shock patients but its predictive value in patients with sepsis and severe sepsis was low. Churpek et al. in a study recommended that qSOFA should not be used as a predictor marker for mortality in patients with suspected infection. ${ }^{13}$ Results of a recently performed meta-analysis defined that qSOFA is a poor sensitive predictor marker of in-hospital mortality in patients with suspected infection. However, the sensitivity of qSOFA was higher for patients in ICUs compared to the patients in other wards. ${ }^{20}$ So, based on the results of these studies and the importance of early detection and appropriate management of septic patients for their outcome, we should emphasis on the following subjects: 1) the pre-hospital and emergency triage based on clinical signs that are easy to recognize and implementation of recommended bundles, 2) the education and implementation of qSOFA score in all medical staff not only intensivists for early detection and appropriate treatment of critically ill patients. Results of another recently published meta-analysis ${ }^{21}$ defined that SIRS criteria were significantly superior to the qSOFA in diagnosis of sepsis, but the qSOFA was slightly better than the SIRS in predicting hospital mortality which is similar to our results. They recommended the combination of both criteria to produce a better model for initiation or escalation therapy in septic patients. ${ }^{22}$ Finkelsztein et al. showed that qSOFA had greater accuracy than SIRS criteria in prediction of ICU free days and mortality. However, results regarding ventilator free days and organ dysfunction free days were inclonclusive. ${ }^{22}$ Haydar and coworkers showed that as a screening tool in emergency department qSOFA needs significantly longer time than SIRS criteria for sepsis detection; thus, relying on qSOFA alone may delay initiation of interventions need for sepsis outcome improvement. ${ }^{8}$ This is in contrast to our results because we used an education course for our medical staff in emergency department regarding qSOFA warning alarms; moreover, we screened patients frequently. Therefore, we identified septic patients earlier compared to the similar trials. Results of JAAMSRA study showed that qSOFA had a suboptimal level for prediction in outside of ICU and could not identify $16 \%$ of patients with sepsis of whom $15.9 \%$ died. ${ }^{23}$ Our results showed that qSOFA could not detect sepsis in almost $35 \%$ but only $5 \%$ of them expired. The lower mortality rate in our trials can be due to early detection of sepsis and appropriate management of them accordingly.

Limitations of the study: Our study was a singlecenter study with almost low sample size in a heterogenous critically ill patients group with infection in a tertiary university affiliated hospital. Thus, we need more trials with larger sample size for generalisability of the results. Strength of our study was implementation of an education course before study and emphasis on frequent screening of patients with suspected infection. The compliance of medical staff with the criteria was so high that lead to early detection and management of these patients which was the strength of our study.

\section{CONCLUSION}

In patients with suspected sepsis, qSOFA has acceptable value for risk stratification of severity, multi organ failure and mortality. It seems that education of medical staff and frequent screening of patients for warning signs can help to increase the value of qSOFA in prediction of mortality in critically ill septic patients. Since the qSOFA criteria is an easy way of assessment with no need for laboratory tests, it can help the pre-hospital caregivers and ED clinicians to identify septic patients at a greater risk of poor outcome. We hope this small study will provoke more investigation into the appropriateness of fully adopting the qSOFA score as a sepsis screening tool by emergency medicine clinicians.

\section{Declaration of interest: None.}

\section{REFERENCES}

1. Cabrita JA, Pinheiro I, Menezes Falcao L. Rethinking the concept of sepsis and septic shock. Eur J Intern Med. 2018;54:1-5. doi: 10.1016/j.ejim.2018.06.002

2. Wang HE, Jones AR, Donnelly JP. Revised national estimates of emergency department visits for sepsis in the United States. Crit Care Med. 2017;45:1443-1449. doi: 10.1097/ CCM. 0000000000002538

3. Singer M, Deutschman CS, Seymour CW, Shankar-Hari M, Annane D, Bauer M, et al. The third international consensus definitions for sepsis and septic shock (Sepsis-3). JAMA. 2016;315(8):801-810. doi: 10.1001/jama.2016.0287 
4. Freund $\mathrm{Y}$, Lemachatti N, Krastinova E, Van Laer M, Claessens YE, Avondo A, et al. Prognostic Accuracy of sepsis-3 Criteria for In-Hospital Mortality among patients with suspected infection presenting to the emergency department. JAMA. 2017;317:301-308. doi: 10.1001/ jama.2016.20329

5. Raith EP, Udy AA, Bailey M, McGloughlin S, MacIsaac C, Bellomo R, et al. Prognostic Accuracy of the SOFA Score, SIRS Criteria, and qSOFA score for In-hospital mortality among adults with suspected infection admitted to the Intensive Care Unit. JAMA. 2017;317:290-300. doi: 10.1001/ jama.2016.20328

6. Peirovifar A, Eydi M, Mirinejhad MM, Mahmoodpoor A, Mohammadi A, Golzari SE. Comparison of postoperative complication between Laryngeal Mask Airway and endotracheal tube during low-flow anesthesia with controlled ventilation. Pak J Med Sci. 2013;29(2):601-605. doi: 10.12669 / pjms.292.2980

7. Rodriguez RM, Greenwood JC, Nuckton TJ, . Comparison of qSOFA with current emergency department tools for screening of patients with sepsis for critical illness. Emerg Med J. 2018;35(6):350-356. doi: 10.1136/ emermed-2017-207383

8. Haydar S, Spanier M, Weems P, Wood S, Strout T. Comparison of QSOFA score and SIRS criteria as screening mechanisms for emergency department sepsis. Am J Emerg Med. 2017;35(11):1730-1733. doi: 10.1016/j.ajem.2017.07.001

9. Maitra S, Som A, Bhattacharjee S. Accuracy of quick Sequential Organ Failure Assessment (qSOFA) score and systemic inflammatory response syndrome (SIRS) criteria for predicting mortality in hospitalized patients with suspected infection: a meta-analysis of observational studies. Clin Microbiol Infect. 2018;pii:S1198-743X(18)30294-5. doi: 10.1016/j.cmi.2018.03.032

10. Simpson SQ. New sepsis criteria: a change we should not make. Chest. 2016;149:1117-1118. doi: 10.1016/j. chest.2016.02.653

11. Cortes-Puch I, Hartog CS. Opening the debate on the new sepsis definition. Change is not necessarily progress: revision of the sepsis definition should be based on new scientific insights. Am J Respir Crit Care Med. 2016;194:1618. doi: 10.1164/rccm.201604-0734ED

12. Moskowitz A, Andersen LW, Cocchi M, Donnino MW. The misapplication of severity-of-illness scores toward clinical decision making. Am J Respir Crit Care Med. 2016;194:256258. doi: 10.1164/rccm.201605-1005ED

13. Churpek MM, Snyder A, Han X, Sokol S, Pettit N, Howell $\mathrm{MD}$, et al. Quick Sepsis related Organ Failure Assessment, Systemic Inflammatory Response Syndrome and Early Warning Scores for Detecting clinical deterioration in infected patients outside the Intensive Care Unit. Am J Respir Crit Care Med. 2017;195(7):906-911. doi: 10.1164/rccm.201604-0854OC

14. Dellinger RP, Levy MM, Rhodes A, Annane D, Gerlach H, Opal SM, et al. Surviving sepsis campaign: international guidelines for management of severe sepsis and septic shock: 2012. Crit Care Med. 2013;41(2):580-637. doi: 10.1097/ CCM.0b013e31827e83af

15. Baig MA, Sheikh S, Hussain E, Bakhtawar S, Subhan Khan $\mathrm{M}$, Mujtaba S, et al. Comparison of qSOFA and SOFA score for predicting mortality in severe sepsis and septic shock patients in the emergency department of a low middle income country. Turkish J Emerg Med. 2018;18(4):148-151. doi: $10.1016 /$ j.tjem.2018.08.002
16. Huson MAM, Katete C, Chunda L, Ngoma J, Wallrauch C, Heller $\mathrm{T}$, et al. Application of the qSOFA score to predict mortality in patients with suspected infection in a resource-limited setting in Malawi. Infection. 2017;45(6):893-896. doi: 10.1007/ s15010-017-1057-5

17. Keeley A, Hine P, Nsutebu E. The recognition and management of sepsis and septic shock: a guide for nonintensivists. Postgrad Med J. 2017;93:626-634.

18. Rudd KE, Seymour CW, Aluisio AR, Augustin ME, Bagenda DS, Beane A, et al. Association of the Quick Sequential (Sepsis-Related) Organ Failure Assessment (qSOFA) Score With Excess Hospital Mortality in Adults With Suspected Infection in Low and Middle-Income Countries. JAMA. 2018;319(21):2202-2211. doi: 10.1001/jama.2018.6229

19. Askim A, Moser F, Gustad TL, , stene H, Gundersen M, Asvold $\mathrm{BO}$, et al. Poor performance of quick-SOFA (qSOFA) score in predicting severe sepsis and mortality - a prospective study of patients admitted with infection to the emergency department. Scand J Trauma Resusc Emerg Med. 2017;25:56. doi: 10.1186/s13049-017-0399-4

20. Maitra S, Som A, Bhattacharjee S. Accuracy of quick Sequential Organ Failure Assessment (qSOFA) score and systemic inflammatory response syndrome (SIRS) criteria for predicting mortality in hospitalized patients with suspected infection: a meta-analysis of observational studies. Clin Microbiol Infect. 2018;24(11):1123-1129. doi: 10.1016/j.cmi.2018.03.032

21. Serafim R, Gomes JA, Salluh J, Póvoa P. A comparison of the Quick-SOFA and systemic inflammatory response syndrome criteria for the diagnosis of sepsis and prediction of mortality: A systematic review and meta-analysis. Chest. 2018;153(3):646655. doi: 10.1016/j.chest. 2017.12.015

22. Finkelsztein EJ, Jones DS, Ma KC, Pabon MA, Delgado T, Nakahira $\mathrm{K}$, et al. Comparison of qSOFA and SIRS for predicting adverse outcomes of patients with suspicion of sepsis outside the intensive care unit. Crit Care. 2017;21(1):73. doi: 10.1186/s13054-017-1658-5

23. Umemura Y, Ogura H, Gando S, Kushimoto S, Saitoh D, Mayumi T, et al. Assessment of mortality by qSOFA in patients with sepsis outside ICU: A post hoc subgroup analysis by the Japanese Association for Acute Medicine Sepsis Registry Study Group. J Infect Chemother. 2017;23(11):757-762. doi: 10.1016/j.jiac.2017.07.005

\section{Authors' Contribution:}

KS: Design of the study, reviewed the final draft.

RJ: Helped in data collection and analysis.

PM: Assisted in analysis and drafting.

AM: Performed the analyses and drafted the manuscript, takes responsibility for integrity of research.

All authors read and approved the final version of the manuscript. 